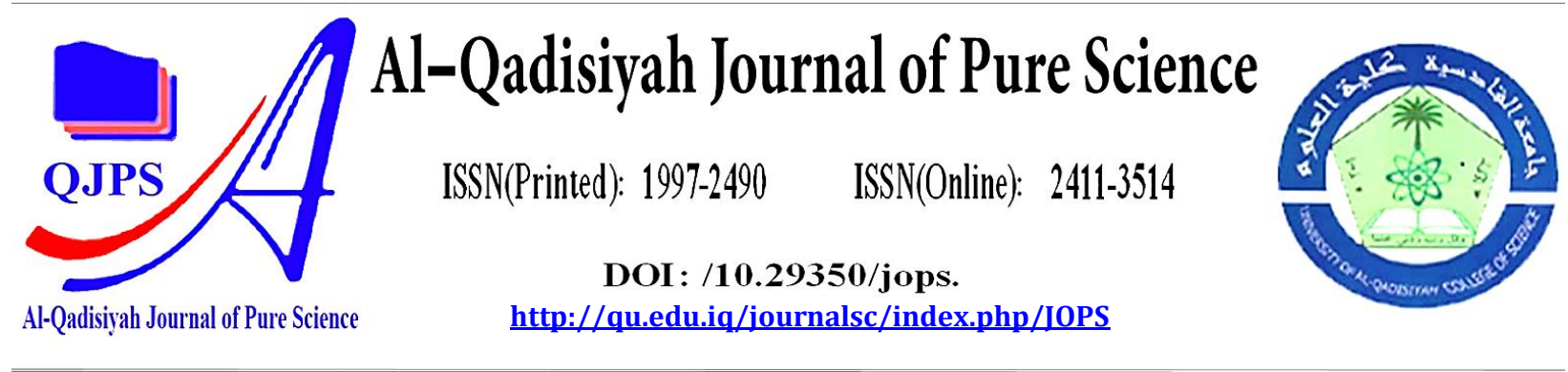

\title{
Estimation of tail parameter for Geometric Brownian motion
}

\section{Authors Names \\ a. Muhannad F. Al-Saadony \\ b. Noor Abd Hassan}

\section{Article History}

Received on: $12 / 8 / 2021$

Revised on: 5/9/2021

Accepted on: 9/9/2021

Keywords:

Heavy-tailed;

Geometric Brownian Motion;

Tail index; Hill estimator;

Bootstrap; Double Bootstrap

and Direct method

DOI: https://doi.org/10.29350/ jops.2021.26. 5.1440

\section{ABSTRACT}

Right-tailed distributions are very important in many applications. There are many studies estimating the tail index. In this paper, we will estimate the tail parameter $(\alpha)$ using the three (Direct, Bootstrap and Double Bootstrap) methods. Our aim is to illustrate the best way to estimate the $\alpha$-stable with $(0<\alpha<2)$ using simulation and real data for the daily Iraqi financial market dataset.

\section{Introduction}

The tail index is the shape parameter of heavy - tailed distributions. Distributions are said to be heavy tailed if the variance is infinite, or the central limit theorem does not work, in which case quintiles and order statistics are used. The Hill estimator ${ }^{[17]}$ is one of the most widely used tools to infer the tail behavior of a distribution, but sometimes this estimator produces poor results. To get rid of the problem of the large bias of the Bootstrap method, Hall in $1990^{[15]}$ suggested using samples with a smaller size than the original sample size, provided that the sample size is very large and the second order parameter $(\boldsymbol{\rho})$ is known. Later, Drees \& Kaufmann ${ }^{[7]}$ proposed a sequential approach to constructing a consistent estimator $\left(\boldsymbol{k}_{n}^{\text {opt }}\right)$ that works asymptotically without knowing the underlying distribution function. To obtain a consistent estimator for the optimal number of order statistics that do 
not need restriction on the parameter $(\boldsymbol{\rho})$, (Danielsson et al., 2001) ${ }^{[5]}$ used a combination of subsample Bootstrap estimates of the difference between two estimators based on Bootstrap sample sizes of different order. (Gomes et al., 2002) ${ }^{[12]}$ introduced a class of semi-parametric estimators for the parameter $(\boldsymbol{\rho})$ with a regularly varying tail and demonstrated that this parameter plays an important role when dealing with optimization problems in extreme values statistics. (Ciuperca \& Mercadier, $2010)^{[4]}$ generalized many studies on the extreme value theory to estimate the extreme value index and the second order parameter $(\boldsymbol{\rho})$. By performing some numerical calculations, the asymptotic normality and consistency were proven under classical assumptions. (Hashemifard et al., 2016) ${ }^{[16]}$ focused on the heavy-tailed stochastic signals generated by the autoregressive continuous-time model, and our aim was to estimate a distribution parameter related to a stable continuous-time autoregressive process with $\mathbf{0}<\alpha<2$. Improving the performance of the Hill estimator has been the goal of many recent publications. For example, (Nemeth, 2020) ${ }^{[24]}$ presented new methods that combine the advantages of the Bootstrap and Kolmogrov-Smirnov approach, where showed that his estimators are able to estimate the parameters of the large tail index, as well as relatively small sample sizes.

In this paper, we generated data used a Geometric Brownian Motion (G.B.M.) model, which is a famous example of Stochastic Differential Equation (S.D.E.). Our aim is to estimate the right- tail parameter using the Direct, Bootstrap and Double Bootstrap methods and then compare the three methods using the mean square errors.

The rest of this article is arranged as follows: Section 2 introduces the heavy tail distributions and their types. Stochastic differential equations and Geometric Brownian motion are presented in section 3. In section 4, we have presented our methods (the Direct, Hill estimator, the Hall 's Bootstrap and the Double Bootstrap) methods. In section 5, the simulation will be presented for our methods. Finally the conclusions are in section 6.

\section{2: Heavy-tailed distributions}

In probability theory, the heavy - tailed distribution is a probability distribution that is not significantly restricted $^{[1]}$. Heavy tail distributions tend to have many extreme values as there will be more density under the p.d.f. curve. The distributions may be heavy right- tailed or left- tailed, or both. In this paper, we have focused on the heavy right- tailed distribution. 


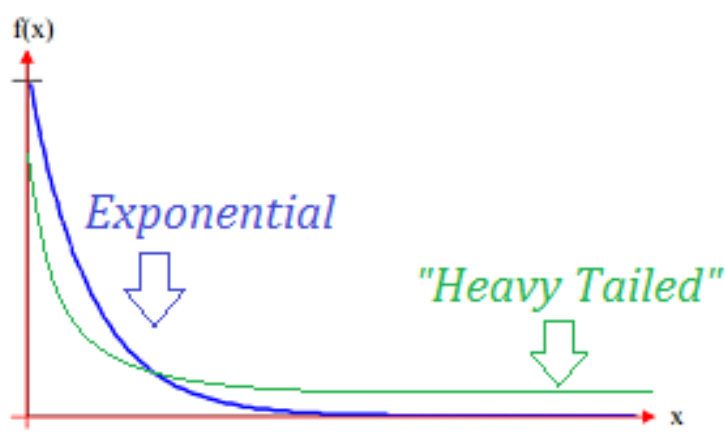

Figure1: the heavy -tailed distribution

Figure1: graph shows the right-heavy tail distribution.

The term heavy tail can be used for distributions whose variance is infinite or whose moments are not limited.

\section{Definition}

The distribution function $\mathrm{F}(\mathrm{X})$ is said to have a (right) heavy tailed with tail index $(\alpha)$ if it satisfies that $^{[25]}:-$

$$
\lim _{t \rightarrow \infty} \frac{1-F(t x)}{F(t)}=x^{-\alpha} \quad \text { for all } x>0 \text { and } \alpha>0
$$

\section{Where:}

$\mathbf{t}$ : is the time

$\mathbf{x}$ : is the random variable

$\mathbf{F}(\mathbf{t})$ : the cumulative function

\section{Definition}

A distribution of the random variable (r.v.) $\mathrm{x}$ with distribution function ( $\mathrm{F}$ ) is said to have a heavy (right) tail if the moment generating function $M_{X}(t)$ of $\mathrm{x}$ is infinite ${ }^{[10][27]}$ i.e.,

$$
\int_{-\infty}^{\infty} e^{t x} d F(x)=\infty \quad \text { for all } t>0
$$

There are three types of heavy-tailed distributions as follows :-

\section{2:1- Fat-tailed distribution}


A fat - tailed distribution is a probability distribution that exhibits a large skewness or kurtosis relative to the normal and exponential distribution. It is a tailed distribution with infinite variance. Many authors state that this type of distribution is a probability distribution with a tail that appears to be fatter than usual. A common example of a fat - tailed distribution is the Log-normal distribution ${ }^{[2]}$.

\section{2:2- Long-tailed distribution}

A distribution of the random variable $(\mathrm{x})$ with distribution function $(\mathrm{F})$ is said to have a long right tailed if ${ }^{[1]}:-$

$$
\lim _{x \rightarrow \infty} \operatorname{Pr}\{X>x+t \mid X>x\}=1 \quad \text { for all } t>0
$$

This means that if the long-tailed quantity exceeds a high level, the probability approaches 1 .

\section{2:3- Sub - exponential distributions}

The distribution that the largest value in the sample contributes the most to the total ${ }^{[22]}$. The properties of heavy tailed distribution as follows:

1- The central limit theory works misleading.

2 - Order statistics are used because some moments do not exist.

\section{3: Stochastic Differential Equations}

Stochastic Differential Equations (SDEs) are used to model many different phenomena such as unstable stock prices and physical systems. These equations contain a variable that is random white noise which is computed as a derivative of Brownian motion. Itô lemma helps to find a solution for SDEs ${ }^{[18][20]}$.

The general form of SDE is ${ }^{[11]}:-$

$$
d x_{t}=\mu d t+\sigma d w_{t}
$$

Where:-

$d x_{t}$ : the change of $x_{t}$ in a continuous time $\mathrm{t}$.

$\mu$ : the drift parameter.

$\sigma$ : the volatility parameter. 
$w_{t}:$ Standard Brownian motion.

A standard Brownian motion is a continuous time and continuous space stochastic process that describes the process of evolution of the value of any random variable. It is sometimes called the Wiener process ${ }^{[19]}$.

We will present the Geometric Brownian Motion as a popular example of SDEs.

\section{3:1.Geometric Brownian Motion}

The Geometric Brownian Motion (GBM), also called the exponential Brownian motion is a continuous- space and continuous-time stochastic process. many Economists prefer the Geometric Brownian motion as a simple model of market prices, because it is positive everywhere (with probability 1) ${ }^{[8]}$. It is an important example of SDEs as it is used to model stock prices in mathematical finance which is called Black and Scholes ${ }^{[18][23]}$. It is modeled by Fisher Black and Myron Scholes ${ }^{[9]}$.

The general form of G.B.M. is ${ }^{[11]}$ :-

$$
d s_{t}=\mu S_{t} d_{t}+\sigma S_{t} d w_{t}
$$

The analytical solution is:

$$
S_{t+1}=S_{t} \exp \left(\left(\mu-\frac{1}{2} \sigma^{2}\right) \Delta T+\sigma D W_{t}\right)
$$

It is easier way to work with the daily returns:

$$
y_{t}=\log \frac{s_{t}}{s_{0}}
$$

Where:

$S_{0}$ is an initial value.

Note that the Geometric Brownian Motion has a log-normal distribution with drift $\mu$ and volatility $\sigma^{[8]}$. 


\section{4: Tail index estimation}

There are many important estimators of the tail index $(\alpha)$, but in this paper we will use the Hill estimator to address the important problems and solutions for estimating the tail index, such as goodness-of-fit test and optimal choice of the sample fraction $(\mathrm{k})^{[25]}$.

\section{4:1. Hil estimator}

It is an important estimator used to detect the presence of heavy tails. To define a Hill estimator we assume that there are non-negative $\left(X_{1}, X_{2}, \ldots \ldots, X_{n}\right)$ observations. . For $1 \leq i \leq n$, write $X_{i}$ for the $\mathrm{i}$ th largest value of $\left(\mathrm{X}_{1}, \mathrm{X}_{2}, \ldots \ldots, \mathrm{X}_{\mathrm{n}}\right)$, so

$$
\mathbf{X}_{1} \leq \mathbf{X}_{2} \leq \cdots \leq \mathbf{X}_{\mathbf{n}}
$$

Then Hill's estimator of $(\alpha)$ is defined as ${ }^{[17]}:-$

$$
\widehat{\alpha}=\left[\frac{1}{k} \sum_{i=1}^{k} \log \frac{x_{n, n-i+1}}{x_{n, n-k}}\right]^{-1}
$$

Where:-

$\mathrm{K}$ :- number of upper-order statistics.

$\hat{\alpha}:$ tail index estimator. It is a consistent estimator for the tail index if the following are achieved ${ }^{[21]}$ :-

$$
\boldsymbol{k} \rightarrow \infty \quad \text { and } \frac{k}{n} \rightarrow 0 \text { as } n \rightarrow \infty
$$

If $\mathrm{k}$ is too large, the estimator is biased, while using a small $\mathrm{k}$ results in a large variance. So, the Hill estimator is strongly dependent on the optimal k selection ${ }^{[6][13]}$.

\section{2:Direct estimation method}

A simple method for choosing optimal $\mathrm{k}$ in the equation (6) directly ${ }^{[25]}$ :

$$
K_{o p t}=\operatorname{argmin}_{k}\left[\frac{\alpha^{4}}{(1+\rho)^{2}} \beta^{2}\left(\frac{k}{n}\right)^{2 \rho}+\frac{\alpha^{2}}{k}\right]
$$

Where: 
$\alpha:$ is the Hill estimator mentioned in (5)

$\mathrm{K}$ : represent the equation (7)

n: sample size

$\rho$ and : are the second order regular variation parameters. It can be calculated using the following steps:-

a) steps to calculate $\rho^{[14]}$ :

$1-$

$$
\begin{gathered}
k=\min \left[n-1,\left(\frac{2 n}{\ln \ln n}\right)\right] \\
S_{n}^{(\theta)}(k)=\left[\frac{1}{k} \sum_{i=1}^{k} \ln \frac{X_{n, n-i+1}}{X_{n, n-k}}\right]^{\theta}
\end{gathered}
$$

(8) $\theta=1,2,3,4$

3- if $l=0$

$$
L_{(n)}^{(l)}(k)=\left[\frac{\ln \left(s_{n}^{(1)}(k)\right)-\frac{1}{2} \ln \left(s_{n}^{(2)}(k) / 2\right)}{\frac{1}{2} \ln \left(S_{n}^{(2)}(k) / 2\right)-\frac{1}{3} \ln \left(S_{n}^{(3)}(k) / 6\right)}\right]
$$

4- if $\rho<0$

$$
\hat{\rho}_{(\mathrm{k})}=-\left|\frac{3\left(\mathrm{~L}_{\mathrm{n}}^{(l)}(\mathrm{k})-1\right)}{\mathrm{L}_{\mathrm{n}}^{(l)}(\mathrm{k})-3}\right|
$$

b) we can calculate $\beta$ as follows ${ }^{[3]}$ :

$$
\hat{\beta}_{\tau}(k)=-\frac{2\left(2+\widehat{\rho}_{k}\right)}{l \widehat{\rho}_{k} \widehat{\alpha}_{k}}\left(\frac{n}{k}\right) \hat{\rho}_{k} \frac{\left[\left(s_{n}^{(1)}(k)\right)^{l}-\left(s_{n}^{(2)}(k) / 2\right)^{\frac{l}{2}}\right]^{2}}{\left(s_{n}^{(2)}(k) / 2\right)^{l}-\left(s_{n}^{(4)}(k) / 24\right)^{\frac{l}{2}}}
$$

Where $\hat{\alpha}_{(k)}, \hat{\rho}_{(\mathrm{k})}$ and $\hat{\beta}_{\tau}(k)$ are consistent estimators for $\alpha, \rho$ and $\beta$ respectively

\section{3:Hall's Bootstrap method}

It is a method used to estimate the parameter of the tail index by sampling a data set with replacement. Hall in1990 suggested the Bootstrap method for estimating the Mean Square Error (MSE) and select smoothing parameter in nonparametric methods. Suppose $\left[X_{1}, X_{2}, \ldots, X_{n}\right]$ denote observations from the distribution function $\mathrm{F}$ and assume ${ }^{[15]}$ :

$$
1-F(X) \sim C X^{-\alpha} \quad C \text { and } \alpha>0
$$




\section{Where:}

c: constant

$\alpha$ : tail index

We wish to estimate $(\boldsymbol{\alpha})$ by using Hill estimator in equation(5) ${ }^{[17]}$. Let $\left[X_{n 1} \geq X_{n 2} \geq \cdots \geq X_{n n}\right]$ denote the order statistics of $\left[x_{n}\right]$ and $[\mathrm{k}]$ is a smoothing parameter. We will choose[ $\left.\mathrm{k}\right]$ to minimize mean square error (MSE) of $\hat{\alpha}^{[15]}$. Let

$$
\operatorname{MSE}(\mathbf{n}, \mathbf{k})=\mathbf{E}[\widehat{\alpha}(K)-\alpha]^{2}
$$

Where:-

$\hat{\alpha}(K):$ is the Hill estimator.

To choose (k) we do the following steps:-

Draw a resample $x_{n 1}^{*}=\left\{x_{1}^{*}, x_{2}^{*}, \ldots \ldots x_{n 1}^{*}\right\}$ from $x_{n} ; n_{1} \leq n$. Let $\left\{x_{n 1,1}^{*} \geq x_{n 1,2}^{*} \geq \cdots \geq x_{n 1, n 1}^{*}\right\}$ denote the order statistics of $\left\{x_{1}^{*}, x_{2}^{*}, \ldots \ldots x_{n 1}^{*}\right\}$, and let ${ }^{[25]}$ :

$$
\widehat{\alpha}^{*}(n 1, k 1)=\left[\frac{1}{k} \sum_{i=1}^{k 1} \log x_{n 1, n 1-i+1}^{*}-\log x_{n 1, n 1-k 1}^{*}\right]^{-1}
$$

Then, the Bootstrap estimate of $\operatorname{MSE}\left(n_{1}, k_{1}\right)$ is:-

$$
\widehat{M S E}\left(n_{1}, k_{1}\right)=E\left[\left\{\widehat{\boldsymbol{\alpha}}^{*}\left(n_{1}, k_{1}\right)-\widehat{\alpha}(n, k)\right\}^{2} \mid x_{1}, x_{2}, \ldots \ldots, x_{n}\right]
$$

Then choose $\hat{k}_{1}$ to minimize $\widehat{M S E}\left(n_{1}, k_{1}\right)$. the optimal is:

$$
\boldsymbol{k}_{\text {opt }}=\boldsymbol{c n}^{y}
$$

for a known $\mathrm{y} \in(0,1)$ but an unknown $c>0$, Hall ${ }^{[15]}$ proposed to estimate $k_{\text {opt }}$ by:

$$
\widehat{\boldsymbol{k}}_{H}=\widehat{\boldsymbol{k}}_{1}\left(\frac{n}{n 1}\right)^{y}
$$




\section{4: Double bootstrap method}

Double Bootstrap method is one of the most accurate estimates which was introduced by ${ }^{[5]}$ and improved by ${ }^{[26]}$. It presents a solution for selecting the sample fraction by the two-step sub-sample Bootstrap method. Then, we reduces the asymptotic mean square error Q(n,k) instead of MSE $(\mathrm{n}, \mathrm{k})^{[25]}$, where:

$$
Q(n, k)=E\left(\frac{1}{2} \widehat{\alpha}^{2}(k)-\widehat{M}(k)\right)^{2}
$$

Where

$$
\widehat{M}(k)=\left\{\frac{1}{k} \sum_{i=1}^{k}\left(\log \frac{X_{n, n-i+1}}{X_{n, n-k}}\right)^{2}\right\}^{-1}
$$

We will explain the steps of this method as follows:

- Draw a resample $\left[X_{1}^{*}, \ldots \ldots, X_{n 1}^{*}\right]$ from $\left[X_{1}, \ldots \ldots, X_{n}\right]$ with $n_{1}=O\left(n^{1-\delta}\right)$ for some $\delta \in\left(0, \frac{1}{2}\right)$.

- $\quad$ Determine the estimators of $\hat{\alpha}(k)$ and $\widehat{M}(k)$ based on the bootstrap sample as $\widehat{\alpha}^{*}(k)$ and $\widehat{M}^{*}(k)$, and choose:

$$
\widehat{k}_{1}=\arg \min _{k 1} E\left\{\left(\frac{1}{2}\left(\widehat{\alpha}^{*}\left(k_{1}\right)\right)^{2}-\widehat{M}^{*}\left(k_{1}\right)\right)^{2} \mid x_{1}, \ldots \ldots, x_{n}\right\}
$$

- $\quad$ Repeat the equation (16) with $\left(n_{2}=\frac{n_{1}^{2}}{n}\right)$ and we get $\hat{k}_{2}$.

- The optimal $k_{o p t}$ is:

$$
\widehat{\boldsymbol{k}}_{D H P V}=\frac{\widehat{\kappa}_{1}^{2}}{\widehat{K}_{2}}\left\{\frac{\left(\log \widehat{k}_{1}\right)^{2}}{\left(2 \log n_{1}-\log \widehat{k}_{1}\right)^{2}}\right\}^{\frac{\log n_{1}-\log \widehat{k}_{1}}{\log n_{1}}}
$$

\section{5: Simulation study}

We have set $(\mathrm{N}=50, \mathrm{~N}=100, \mathrm{~N}=150, \mathrm{~N}=200$ and $\mathrm{N}=250)$. We have generated Geometric Brownian motion driven Brownian motion. Then we have get tail index $(\alpha), K_{o p t}$ and the mean square error (MSE) for each sample. We let $\mu=0.5$ and $\sigma=0.05$. The data is simulated using equation (4). 
Table 5.1: $\alpha$, MSE and $K_{o p t}$ for five samples based on 100 replications.

\begin{tabular}{|c|c|c|c|}
\hline $\mathrm{N}=50$ & Bootstrap & Double Bootstrap & Direct \\
\hline$\alpha$ & 0.0102245247 & 0.033000000 & 0.07107605 \\
\hline MSE & 0.0001292511 & 0.001716803 & 0.01004159 \\
\hline$K_{o p t}$ & 3.0000000000 & 2.000000000 & 7.11581631 \\
\hline \multicolumn{4}{|c|}{$\mathrm{N}=100$} \\
\hline$\alpha$ & 0.0105181566 & 0.0223157895 & 0.041829245 \\
\hline MSE & 0.0001373524 & 0.0004991821 & 0.002247387 \\
\hline$K_{\text {opt }}$ & 3.0000000000 & 2.0000000000 & 0.490268148 \\
\hline \multicolumn{4}{|l|}{$\mathrm{N}=150$} \\
\hline$\alpha$ & 0.0162245871 & 0.045960000 & 0.04216307 \\
\hline MSE & 0.0003937763 & 0.002791125 & 0.00261230 \\
\hline$K_{o p t}$ & 3.4100000000 & 2.610000000 & 0.25461379 \\
\hline \multicolumn{4}{|l|}{$\mathbf{N}=\mathbf{2 0 0}$} \\
\hline$\alpha$ & 0.0164169738 & 0.054640000 & 0.050240318 \\
\hline MSE & 0.0003870336 & 0.003323239 & 0.002988345 \\
\hline$K_{\text {opt }}$ & 3.9500000000 & 2.970000000 & 0.168603382 \\
\hline \multicolumn{4}{|l|}{$\mathbf{N}=\mathbf{2 5 0}$} \\
\hline$\alpha$ & 0.0224340013 & 0.066000000 & 0.056774807 \\
\hline MSE & 0.0008237568 & 0.005643042 & 0.003709766 \\
\hline$K_{\text {opt }}$ & 4.1200000000 & 3.350000000 & 0.041839528 \\
\hline
\end{tabular}

Table (5.1) represent the value of $\alpha$, MSE and $K_{o p t}$ for our model using Direct, Bootstrap and Double Bootstrap methods. When 100 replications, it is obvious that the worst performance is for the direct method. the performance of the Bootstrap method is much better than the others. For all sample sizes, the performance of the Double Bootstrap method was near of the Bootstrap method performance. 
geometric Brownian Motion

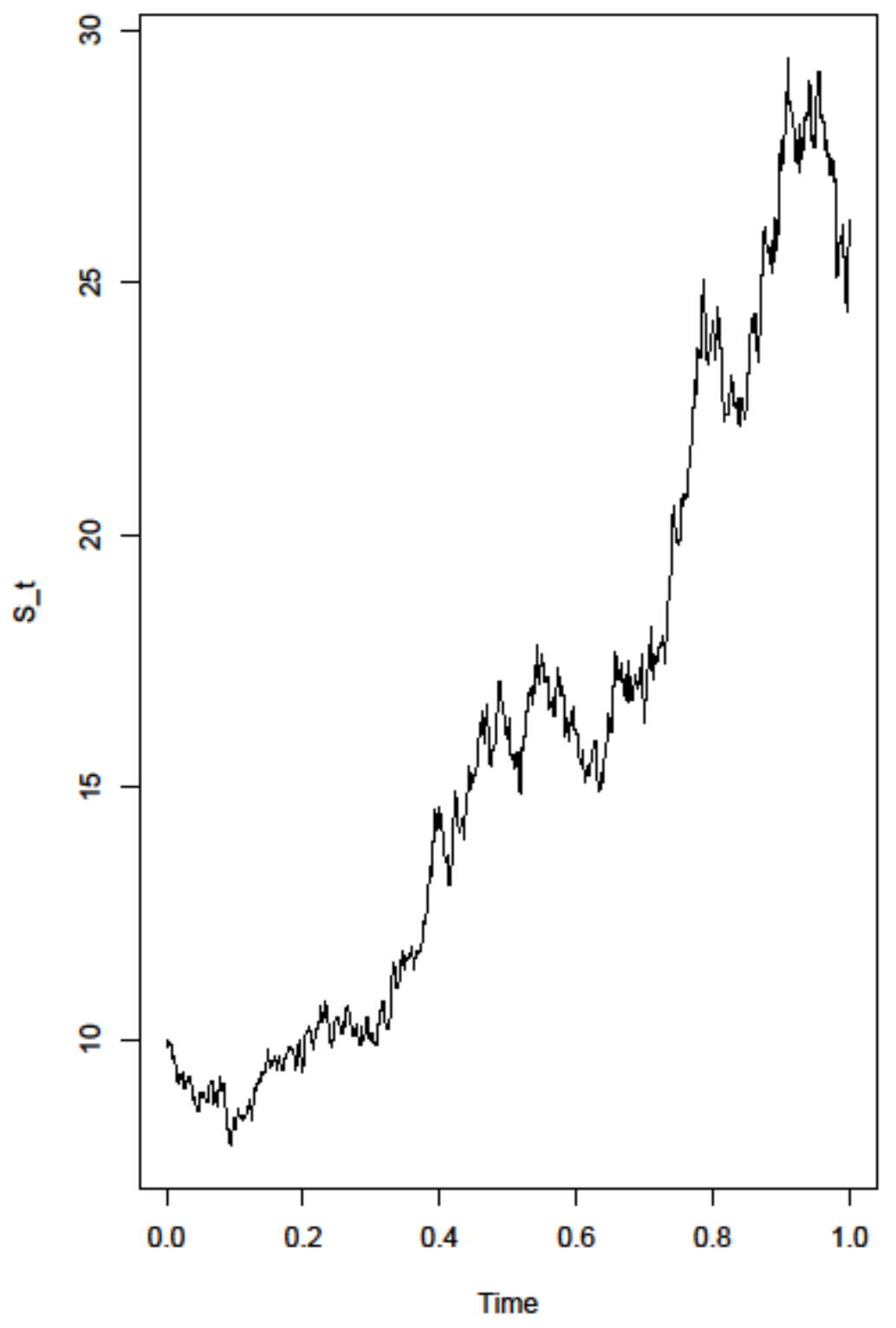

Figure 5.1: the Geometric Brownian Motion model through the time.

Figure (5.1) shows the movement of Geometric Brownian motion through time. It is clearly that the process affect by Brownian motion and always is positive. 
Return of geometric Brownian Motion

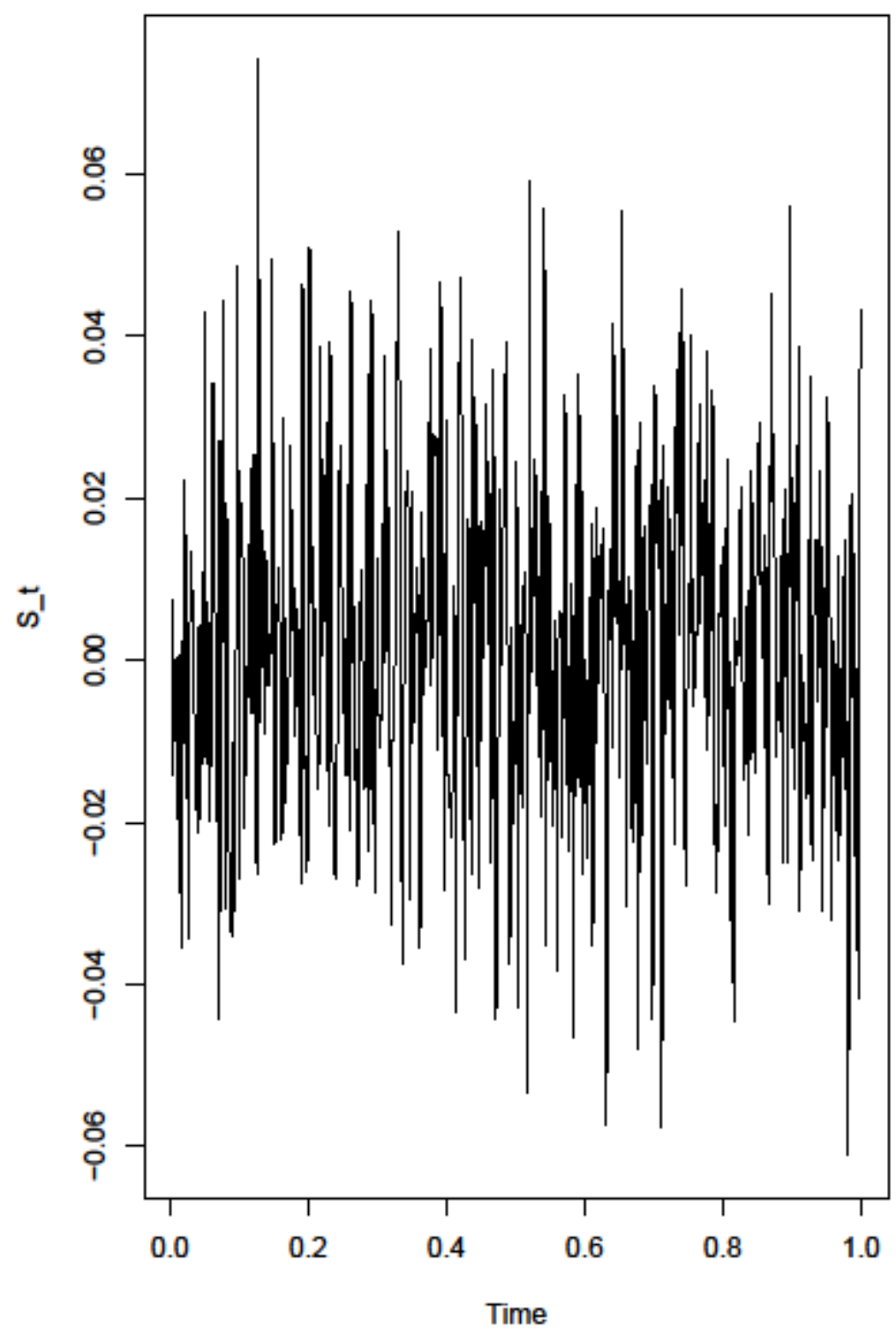

Figure 5.2: the returns through the time.

Figure (5.2) shows the movement of Geometric Brownian motion returns through time. It is clearly that the process affect by Brownian motion and always is positive. 


\section{6: Conclusion}

We have used some nonparametric methods to estimate the tail parameter, for the Stochastic Differential Equation (SDE) such as G.B.M model. From the simulation and based on the results of the MSE, we found that the Bootstrap method for parameter estimation is the best method for estimation, as it achieved the lowest MSE.

\section{References}

1. Asmussen, S. (2003). Steady-State Properties of gi/g/1. Applied probability and Queues, 266301.

2. Bahat, D. , Rabinovich, A. \& Frid, V. (2005). Tensile Fracturing in Rocks (p.570). Springerverlag Berlin Heidelberg.

3. Caeiro, F., \& Gomes, M. I. (2006). A new class of estimators of a "scale" second order parameter. Extremes, 9(3-4), 193-211.

4. Ciuperca, G. \& Mercadier, C. (2010). Semi-parametric estimation for heavy tailed distributions. Extremes, 13(1), 55-87.

5. Danielsson, J., de Haan, L., Peng, L. \& de Vries, C. G. (2001). Using a bootstrap method to choose the sample fraction in tail index estimation. Journal of Multivariate Analysis, 76(2), 226-248.

6. Danielsson, J., Ergun, L. M., de Haan, L. \& de Vries, C. G. (2016). Tail index estimation: Quantile driven threshold selection. Available at SSRN 2717478.

7. Drees, H., \& Kaufmann, E. (1998). Selecting the optimal sample fraction in univariate extreme value estimation. Stochastic Processes and their applications, 75(2), 149-172.

8. Dunbar, S. R. (2016). Stochastic Processes and Advanced Mathematical Finance. The Definition of Brownian Motion and the Wiener Process, Department of Mathematics, 203, 68588-0130.

9. Fisher, B. \& Scholes, M. (1973). The Pricing of Options and Corporate Liabilities. Journal of Political Economy, 6, 637- 654.

10. Foss, S., Korshunov, D. \& Zachary, S. (2011). An Introduction to Heavy-Tailed and Sub exponential Distributions. (vol. 6, pp. 0090-6778). New York: springer.

11. Franke, J., Hardle, W. K. \& Hafner, C. M. (2004). Statistics of Financial Markets ( vol. 2). Berlin: Springer. 
12. Gomes, M. I., De Haan, L. \& Peng, L. (2002). Semi-Parametric Estimation of the Second Order Parameter in Statistics of Extremes. Extremes, 59(4), 387-414.

13. Gomes, M. I., Pestana, D. \& Caeiro, F. (2009). A note on the asymptotic variance at optimal levels of a bias-corrected Hill estimator. Statistics \& Probability Letters, 79(3), 295-303.

14. Gomes, M.I., \& Pestana, D. (2007). A simple second-order reduced bias tail index estimator. Journal of statistical computation and simulation, 77(6), 487-502.

15. Hall, P. (1990). Using the bootstrap to estimate mean squared error and select smoothing parameter in nonparametric problems. Journal of Multivariate Analysis, 32(2), 177-203.

16. Hashemifard, Z., Amindavar, H. \& Amini, A. (2016). Parameters Estimation for ContinuousTime Heavy-Tailed Signals Modeled By $\alpha$-Stable Autoregressive Processes. Digital signal processing, 57, 79-92.

17. Hill, B. M. (1975). A simple general approach to inference about the tail of a distribution. Annals of Statistics, 1163-1174.

18. Iacus, S. M. (2009). Simulation and Inference for stochastic Differential Equation with R Examples. Springer Science \& Business Media.

19. Iacus, S. M. (2011). Option Pricing and Estimation of Financial Model with R. New York: Wiley.

20. Imkeller, P. \& Schmalfuss, B. (2001). The conjugacy of stochastic and random differential equations and the existence of global attractors. Journal of Dynamics and Differential Equations, 13(2), 215-249.

21. Mason ${ }^{\mathrm{a}}$, D. M. (1982). Law of Large Number for Sums of Extreme Values. The Annals of Probability, 754-764.

22. Mikosch, T. (1999). Regular Variation, Sub exponentiality and Their Applications in Probability Theory (vol.99). Eindhoven, the Netherlands: Eindhoven University of Technology.

23. Mikosch, T. (2004). Elementary Stochastic Calculus (5 th ed.).World Scientific, Denmark.

24. Nemeth, L. \& Zempleni, A. (2020). Regression estimation for the tail index. Journal of Statistical Theory and Practice, 14(3), 1-23.

25. Peng, L. \& Qi, Y. (2017). Inference for Heavy-Tailed Data: Applications in Insurance and Finance. Academic press.

26. Qi, Y. (2008). Bootstrap and empirical likelihood methods in extremes. Extremes, 11(1), 81-97. 
27. Rolski, T., Schmidli, H., Scmidt, V. \& Teugels, J. L. (2009). Stochastic Processes for Insurance and Finance. (Vol. 505). John wiley \& Sons. 